\title{
Dank an die Gutachter
}

Herausgeber und Schriftleitung bedanken sich bei den im folgenden genannten Damen und Herren sehr herzlich für die im Jahre 2000 geleistete Begutachtung der ihnen übersandten Manuskripte.

Biscoping J., Karlsruhe

Böck M., Würzburg

Boldt J., Ludwigshafen

Borberg H., Köln

Caspari G., Gießen

Dahr W., Berg. Gladbach

Ebell W., Berlin

Eckstein R., Erlangen

Gathof B., Köln

Gerlich W.H., Gießen

Gombotz H., Graz

Greinacher A., Greifswald

Große-Wilde H., Essen

Gürtler L., Greifswald

Hanfland P., Bonn

Hansen E., Regensburg

Heim M.U., Magdeburg

Hellstern P., Ludwigshafen

Hennig H., Lübeck

Holzberger G., Kassel

Karger R., Marburg

Kiefel V., Rostock
Kirchner H., Lübeck

Kiesewetter H., Berlin

Klüter H., Lübeck

Köhler M., Göttingen

Lassen U., Springe

Mertens T., München

Meßmer K., München

Müller N., Essen

Müller-Berghaus G., Bad Nauheim

Neidhardt B., Erlangen

Pötzsch B., Bonn

Renz H., Marburg

Roelcke D., Heidelberg

Roth W.K., Frankfurt/Main

Salama A.G., Berlin

Schlenke P., Lübeck

Schönitzer D., Innsbruck

Seyfert U.T., Homburg

Sibrowski W., Münster

Singbartl G., Hamburg

Spahn D., Zürich

Stangel W., Hannover 